\title{
European College of Gerodontology - Gerostomatologie heute
}

\begin{abstract}
Die Tagung des European College of Gerodontology findet zur selben Zeit wie der Österreichische Zahnärztekongress, und ebenfalls im Kongresshaus Graz, statt. Welcher Gedanke steckt dahinter? Über 50 Prozent unserer Patienten sind über 50 Jahre alt. Die demographische Entwicklung zeigt, dass der Anteil Betagter und Hochbetagter rasant im Zunehmen begriffen ist. Die Zahnheilkunde muss sich diesen Anforderungen stellen und Konzepte präsentieren, die nicht nur die Prävention bei Jugendlichen im Auge haben, sondern ebenso die besonderen Herausforderungen der Prävention und Therapie der Senioren berücksichtigen.

Vor diesem Hintergrund sind wir besonders glücklich, dem Thema Alterszahnheilkunde im Rahmen des österreichischen Zahnärztekongresses durch die Abhaltung eines internationalen Kongresses einen besonderen Stellenwert geben zu können, um die Kollegenschaft für dieses Thema zu sensibilisieren.
\end{abstract}

\section{Wie ist die Situation der Gerosto- matologie heute in Europa?}

Die Europäische Kommission hat im Jahre 2010 in ihrem Annex V.3/5.3.1 of Directive 2005/36/EC die Alterszahnheilkunde als verpflichtenden Teil der zahnärztlichen Ausbildung aufgenommen. Das Wissen um die Brisanz dieses Themas ist bekannt, aber es ist notwendig über lokale Initiativen hinaus sozialmedizinische Gesamtkonzepte zu entwickeln und umzusetzen.

Wie präsentiert sich Österreich auf dem Gebiet der Gerostomatologie? Wir haben in Graz im Jahre 2006 das erste österreichische Curriculum Alterszahnheilkunde implementiert, welches zum Fortbildungsdiplom Gerostomatologie der österreichischen Zahnärztekammer führt. Wir haben zahlreiche lokale Initiativen durch engagierte Kollegen und Kolleginnen. Was fehlt ist die Umsetzung des vorhandenen Wissenstandes in die sozialmedizinische Gesetzgebung. Wir fordern von Seiten der

\section{Wir müssen zahnärztliche \\ Gesamtkonzepte umsetzen, welche alle Anteile der \\ Bevölkerung umfassen.}

ARGE Gerostomatologie schon seit Jahren, die verpflichtende zahnmedizinische Beurteilung durch den Zahnarzt vor Eintritt in eine Senioreninstitution, die Aufnahme der Zahn- und Mundpflege in die pflegerische Ausbildung, sowie die verpflichtende Aufnahme dieses wesentlichen Punktes in das Pflegeprotokoll.

\section{In welchen Bereichen der Gerosto-} matologie wird derzeit am intensivsten geforscht?

Die Zusammenhänge zwischen Entzündungen in der Mundhöhle und allgemeinmedizinischen Erkrankungen sind evident und dokumentiert. Einer der interessantesten Aspekte, der auch ein Hauptthema des ECG-Kongresses in Graz sein wird, ist der mögliche Zusammenhang von Parodontitis und M. Alzheimer.

Wo sind Ihrer Meinung nach Defizite in der zahnärztlichen Versorgung älterer Menschen in Europa?

Wie bereits angesprochen, gibt es umfangreiche, erfolgreiche Initiativen in Bezug auf Prophylaxe im jugendlichen Alter. Eine entsprechende präventive Versorgung im Alter ist nicht existent.

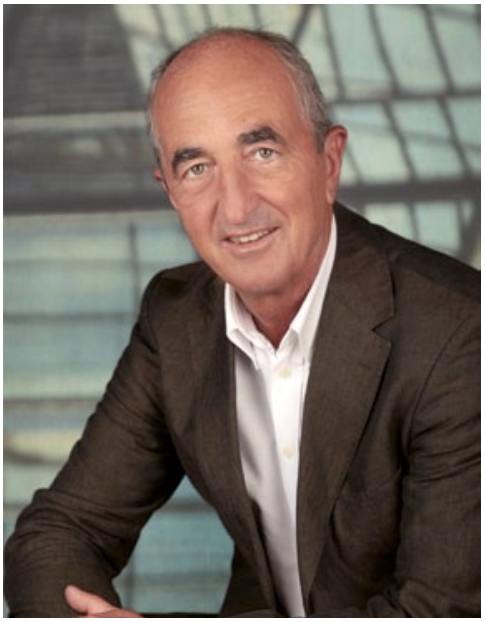

Univ.-Prof. Dr. Gerwin Arnetzl

Wir müssen zahnärztliche Gesamtkonzepte umsetzen, welche alle Anteile der Bevölkerung umfassen.

Wird es Querverbindungen zwischen Ihrem Kongress und dem Österreichischen Zahnärztekongress geben? Mit der Gebühr zum österreichischen Zahnärztekongress haben Sie auch uneingeschränkt die Möglichkeit, die Vorträge am internationalen ECG-Kongress zu besuchen. Wir hoffen, dass zahlreiche Kollegen die Möglichkeit wahrnehmen, unseren hochkarätigen Referenten zu folgen.

\section{Was wünschen Sie sich für Ihren} Kongress?

Wir wünschen uns für unseren Kongress, eine Nachhaltigkeit zu den Themen der Alterszahnheilkunde in Österreich zu erreichen.

\section{Vielen Dank!}

Dr. Renate Höhl, 5. 8. 2013 